\title{
Zukunftstarifvertrag Airbus - Renaissance qualitativer Tarifpolitik?
}

\author{
DANIEL FRIEDRICH
}

Der 7.10.2011 bringt den Durchbruch. 11.000 Beschäftigte von Airbus in Deutschland beteiligen sich an diesem Tag am Warnstreik der IG Metall Küste und legen ihre Arbeit befristet nieder. Sie kämpfen damit für eine langfristige Sicherung ihrer Einkommen, Standorte und Arbeitsplätze. Aber ein wesentlicher Motor der Mobilisierung ist gar nicht die Angst um den Arbeitsplatz oder die Sorge um die Zukunft des Unternehmens. Der Wunsch der Beschäftigen nach mehr Beteiligung bei Entscheidungen, mehr Autonomie am Arbeitsplatz und mehr Respekt vor ihrer Arbeit ist es - das wollen sie durch den Zukunftstarifvertrag verbessert sehen. Zwölf Tage später haben sie es geschafft. Die Airbus-Geschäftsleitung, der Arbeitgeberverband Nordmetall, die IG Metall Küste und der Gesamtbetriebsrat des Unternehmens unterzeichnen ein „Eckpunktepapier zum Zukunftstarifvertrag“. Wesentliche Bestandteile sind eine Beschäftigungs-, Einkommens- und Standortsicherung bis zum 31.12.2020. Darüber hinaus werden die Beteiligungsrechte und die $\mathrm{Au}$ tonomie der Beschäftigten bei ihrer Arbeit gestärkt und die ausufernde Leiharbeit beschränkt. Damit endet nach eineinhalb Jahren die Auseinandersetzung um gute, zukunftssichere Arbeitsplätze und Standorte. Gleichzeitig setzt die IG Metall damit ein Zeichen, dass die Beschäftigten auch für gute Arbeitsbedingungen mobilisierbar und konfliktbereit sind.

\section{Der Vorläufer - „Sicherheit durch Flexibilität“}

Das im Jahre 2003 vereinbarte Vertragswerk „Sicherheit durch Flexibilität“" (SiduFlex) der Arbeitnehmervertreter und des Managements bei Airbus setzte einen neuen Maßstab in den Arbeitsbeziehungen. Dem Grundsatz , arbeitsplatzschaffende Instrumente haben Vorrang vor arbeitszeitverlängernden Instrumenten " folgend, werden Arbeitszeitkonten inklusive Lebensarbeitszeitkonten geschaffen, Flexibilisierungsmaßnahmen ausgeweitet und die damals aufkommende Leiharbeit ab dem vierten Monat bei Bezahlung und Arbeitsbedingungen den Stammbeschäftigten gleichgestellt sowie die Ausbildung auf mindestens $5 \%$ der Belegschaft angehoben und Auslerner unbefristet übernommen. Man will damit die „Politik des kontrollierten Wachstums auch für die kommenden Jahre unterstützen, aber auch für etwaige Rückschläge entsprechende Reaktionsmöglichkeiten schaffen“` Beide Seiten haben bei Vertragsabschluss die Luftfahrtkrise und die Auseinandersetzung um das Sparprogramm „Dolores“ Mitte der 1990er Jahre vor Augen, welches zwar ohne betriebsbedingte Kündigungen auskam, aber trotzdem tausende Arbeitsplätze kostete. Gerade die Umsetzung und Produktion des Großraumflugzeuges A380 stellt das Unternehmen und die Beschäftigten vor eine enorme Herausforderung. Die Zahl der Leiharbeitnehmer steigt extrem an. Aufgrund der Gleichstellung der Leiharbeitnehmer ab dem vierten Monat wird dies ohne große Gegenwehr geduldet. Mit später teilweise über 40 \% Leiharbeitnehmern im Unternehmen - in manchen Unternehmensbereichen sogar über 70 \% - kippt die Stimmung. Als dann im Zuge der Verzögerungen beim A380 das Unternehmen mit dem Sparprogramm „Power8“ reagiert, wird zusätzlich klar, dass die Zeit der Sozialpartnerschaft zwischen Arbeitgeber und den Beschäftigten sowie ihren Vertretern vorerst vorbei ist.

\section{Ein normales Unternehmen?}

„Airbus muss ein normales Unternehmen werden“ - die Aussage der EADS-Vorsitzenden Louis Gallois und Tom Enders im Jahre 2007 beziehen viele ausschließlich auf den Einfluss der Politik auf das Unternehmen. Es ist aber viel mehr gemeint: Anstelle einer konstruktiven, gemeinsamen und auch fortschrittlichen Mitbestimmungskultur soll die Zusammenarbeit mit den Betriebsräten und Gewerkschaften auf das gesetzliche Mindestmaß reduziert werden und das Mitwirken der Arbeitnehmervertreter auf Unternehmensentscheidungen verhindert werden. Mit entsprechenden Neubesetzungen im Management wird dieser neue Weg untermauert. Zudem geht er einher mit einer unkontrollierten Zentralisierung und Orientierung am Ziel „Lean“. Zentrale Vorgaben drangsalieren die persönliche Arbeitsorganisation. Ingenieure entwickeln und forschen nicht mehr selbst, sondern sind manchmal fast ausschließlich mit der Vergabe und Kontrolle von Werkaufträgen beschäftigt. Arbeitspakete werden gegen den ausdrücklichen Rat der Beschäftigten nach außen vergeben - die wegen fehlender Qualität notwendigen Nacharbeiten aus diesen Maßnahmen müssen dann dieselben Beschäftigten leisten. Die Autonomie der Beschäftigten bei der Arbeitszeitgestaltung soll er-

Konzernbetriebsvereinbarung SiduFlex vom 1.4.2003. 
heblich eingeschränkt und die teilautonome Gruppenarbeit abgeschafft werden. Vor lauter Reportauflagen an die Zentrale kommen manche Beschäftigten nicht mehr zur eigentlichen Arbeit. Die Produktion soll in Mini-Factorys umgebaut werden - kapazitätsorientierte Arbeitszeiten inklusive. Kurzum: Die Stimmung ist schlecht, die Führungskräfte versagen, der Krankenstand steigt extrem und die Beschäftigten geben ihrer Führung in einer internen Mitarbeiterbefragung alarmierende Noten. Eine adäquate Reaktion des Unternehmens auf die Ergebnisse bleibt aus. Airbus ist tatsächlich auf dem Weg zu einem normalen Unternehmen läuft aber Gefahr, seine Mitarbeiter dabei zu verlieren.

\section{Von SiduFlex zum Zukunftstarifvertrag}

Nach Abschluss von Power8 greifen die Betriebsräte und die IG Metall diese Stimmung und Probleme auf. Mit Blick auf die EADS-Vision 2020 (u. a. Steigerung der Profitabilität auf eine Umsatzrendite von mindestens $10 \%$, Fokussierung auf das Kerngeschäft, $40 \%$ Wertschöpfung und $20 \%$ Personalanteil außerhalb Europas, etc.) ist klar, die Vereinbarung „Sicherheit durch Flexibilität“ muss über das Ende der Laufzeit zum 31.12.2012 optimiert und verlängert werden. Ein Wandel des Unternehmens geht nur mit den Beschäftigten und diese brauchen dafür Sicherheit und keine Angst um Arbeitsplätze, Einkommen oder Standorte. Darüber hinaus sollen aber auch die „Soft Skills“ angepackt werden. Mit einer Mitarbeiterbefragung, Vertrauensleutegesprächen und -veranstaltungen sowie in Betriebsversammlungen werden Meinungen und Positionen eingeholt. Klares Ergebnis: Sicherheit ist das eine, gute Arbeit das andere. Man will endlich eine Perspektive für die Leiharbeitnehmer, von denen über die Hälfte länger als 24 Monate teilweise über sechs und sieben Jahre - bei Airbus sind. Die Beschäftigten sollen bei der Vergabe von Arbeitspaketen mitbestimmen, die persönliche Autonomie soll gestärkt und die zentrale Ausrichtung der Arbeit zurückgedrängt werden. Mit einer Verknüpfung von sicherer und guter Arbeit gehen IG Metall und Gesamtbetriebsrat in die Gespräche und können sich letztendlich - nach entsprechender Konfliktbereitschaft im Betrieb - in vielen wichtigen Punkten durchsetzen:

(1) Sichere Arbeitsplätze: Einkommen, Beschäftigung und Standorte sind bis Ende 2020 gesichert. Das individuelle Einkommen darf sich durch Auswirkungen von Veränderungsprozessen nicht reduzieren. Die Kernkompetenzen der Standorte müssen erhalten werden und zu deren Erhalt entsprechende Investitionen getätigt werden. Die aktuelle Personalstärke (Stand 31.12.2011) ist als Minimalbestand zu halten. 2016 kann der Arbeitgeber von einer Revisionsklausel Gebrauch machen. Diese hätte allerdings die Beendigung aller Bestandteile des Zukunftstarifvertrages zur Folge, also auch der zusätzlichen Flexibilisierung.

(2) Flexible Arbeit: Die Regelungen von SiduFlex wurden verlängert. Das Unternehmen kann weiter über Arbeitszeit- konten und andere Instrumente auf Auslastungsschwankungen reagieren. Die Beschäftigten behalten weitgehende Autonomie über ihre Arbeitszeiten und können über ein Lebensarbeitszeitkonto früher aus dem Betrieb ausscheiden. Auslastungslücken können über ein kollektives Sicherheitskonto gesteuert werden. Leiharbeit wird - bei Beibehaltung von Equal-Pay ab dem vierten Monat - auf 20 \%, ab 2015 auf $15 \%$ beschränkt. Durch diese Rückführung muss die Stammbelegschaft entsprechend aufgestockt werden (bis zum 31.8.2012 wurden schon über 700 Leiharbeitnehmer fest übernommen). Zudem muss bei einer Beschäftigungsdauer von 24 Monaten eine Festanstellung geprüft werden, allerdings ohne Rechtsanspruch. Für neue Flugzeugprojekte wie den A350 werden bei Bedarf eigenständige Regelungen geschaffen.

(3) Mehr Mitsprache: Grundprinzip der Arbeit bei Airbus ist die selbst organisierte Teamarbeit. Dabei wird die Arbeit weitgehend mit hoher Eigenverantwortung auf Teamebene selbst gestaltet. Dies gilt für den gewerblichen Bereich wie auch für die Arbeitsplätze in der Verwaltung, Forschung und Entwicklung etc. Das bekannte Prinzip der autonomen Gruppenarbeit mit eigener Steuerung und Planung der Arbeit aus der Produktion soll damit im gesamten Unternehmen als Grundsatz verankert werden. Ein wichtiges Merkmal dabei: Die Beschäftigten wählen überall ihre Teamsprecher selbst. Die Beschäftigten und die Betriebsräte sind bei der Vergabe von Arbeitspaketen an Dritte und bei inhaltlichen, räumlichen und organisatorischen Veränderungen in ihrem Arbeitsbereich rechtzeitig und umfassend zu informieren und bei der Umsetzungsplanung verbindlich zu beteiligen. Meinungsverschiedenheiten werden über einen Eskalationsprozess bis hin zur Betriebsratsspitze und Standortleitung ausgetragen. Zwar behält der Arbeitgeber das letztendliche Entscheidungsrecht, allerdings dürfen die Maßnahmen tatsächlich erst nach einer Einigung mit den Beschäftigten oder nach dem letzten Einigungsversuch auf Spitzenebene durchgeführt werden.

(4) Besser statt billiger: Anstelle von Einsparungen durch eine starre Vorgabe von Produktivitätssteigerungen wurde ein befristeter, beteiligungsorientierter Optimierungsprozess vereinbart. In 18 Monaten sollen in allen Bereichen des Unternehmens Ideen zur Verbesserung der Arbeit und der Arbeitsbedingungen und damit auch zur Steigerung der Unternehmensleistung und der Motivation erarbeitet werden. Dabei werden mit allen Beschäftigten in ihren Bereichen Ideenforen durchgeführt, die Ergebnisse gemeinsam bewertet und die Umsetzung wird grundsätzlich im Bereich entschieden. Nur zum Beispiel finanziell schwerwiegende oder Arbeitsbereiche übergreifende Ideen müssen in weiteren Hierarchien entschieden werden. Geregelt sind dabei auch Transparenz über den weiteren Verlauf, die verschiedenen Verantwortungsebenen und die Bereitstellung der nötigen Mittel. 
(5) Gute Ausbildung: Die Ausbildungsquote von $5 \%$ der Stammbelegschaft und die unbefristeten Übernahmen bleiben erhalten. Zusätzlich können mindestens $10 \%$ der Auslerner ihr Arbeitsverhältnis bei Airbus ruhen lassen und im Rahmen eines Studiums in ein eigenes Mentoring-Programm wechseln.

\section{Zwischenfazit}

Ein Jahr nach Abschluss des Eckpunktepapieres und sieben Monate nach Beginn der Umsetzung der Inhalte kann man ein erstes Zwischenfazit ziehen. Mit dem Zukunftstarifvertrag haben die Beschäftigten, die Arbeitnehmervertreter und die IG Metall einen großen Erfolg und einen entsprechenden Kulturwandel bei Airbus durchgesetzt. In der Pilotphase des Optimierungsprozesses haben sich über 2.300 Beschäftigte beteiligt und durchschnittlich hat jeder mindestens eine Idee zur Verbesserung der Arbeit und Abläufe eingebracht. Wer durch die Standorte geht, trifft immer wieder mal auf Veränderungen, die durch diesen Prozess endlich eingeführt wurden. Auf diese sind Beschäftigte besonders stolz und sie erleben endlich einen ernsthaften Umgang mit ihren Meinungen und Ideen. Da dies nicht nur die Arbeit für die Arbeitnehmer vereinfacht, sondern auch die Produktivität steigert, sind die Ergebnisse auch für Airbus von Nutzen. Wer sich mit den Beschäftigten, aber auch mit den Führungskräften unterhält, hört oft die Frage: Warum haben wir das nicht schon früher umgesetzt?

Mit den neuen Regeln bei der Fremdvergabe und bei Veränderungen von Arbeitsabläufen und -inhalten wurde den Beschäftigten ein neues Instrument in die Hand gegeben. Gerade wenn Entscheidungen manchmal transnational erfolgen oder ohne weitere Überprüfung auf Anweisung der Zentrale durchgeführt werden, ist es gut, eigene Informations- und Beteiligungsrechte zu haben, um der Floskel „das ist aber so entschieden worden“ entgegentreten zu können.

Auch das Unternehmen reagiert auf die überzogene Zentralisierung der Vergangenheit und will das Organisationsprinzip von Airbus weltweit verändern. ${ }^{2}$ Hauptaugenmerk liegt dabei auf der Stärkung der Strukturen vor Ort und einer Erhöhung der Entscheidungskompetenz der örtlichen Führungskräfte und Standortleiter. Die Planungen zu einer Fusion zwischen dem Airbus-Mutterkonzern EADS und dem britischen Rüstungskonzern BAE Systems brachten zusätzliche Unruhe. Mit der Beschäftigungs-, Einkommens- und Standortzusage aus dem Zukunftstarifvertrag haben die Beschäftigten die Gewissheit, dass solche Veränderungen nicht zu Verschlechterungen ihrer Arbeitsbedingungen führen.

Selbstkritisch muss man feststellen, dass nicht alle Ziele bei der Begrenzung der Leiharbeit erzielt wurden. Gerade, dass es keine Höchstdauer der Beschäftigung von Leiharbeitskräften gibt und es weiterhin möglich ist, diese ohne eine direkte Perspektive der Übernahme jahrelang bei Airbus

2 Hegmann, G. (2012): Strategiewechsel: Airbus-Werke bekommen mehr Freiheit, in: FinancialTimes Deutschland vom 11.9. einzusetzen, bleibt unbefriedigend. Mit der Höchstquote von 20 bzw. 15 \% und der Beibehaltung der gleichen Behandlung (Monatsentgelt, Sonderzahlungen, betriebliche Leistungen, etc.) wurden zumindest kollektive Perspektiven aufgebaut und ein Mindestmaß an sozialer Sicherheit garantiert.

Die Auseinandersetzung um den Zukunftstarifvertrag hat die IG Metall und die Betriebsräte stärker gemacht. Es konnte ein Rekord bei den Gewerkschaftsbeitritten verzeichnet werden. Dieser Erfolg kam gerade auch aus Bereichen der Hochqualifizierten, die bisher wenig mit der IG Metall als Vertreter ihrer Interessen verbanden. Aber nicht nur quantitativ konnte man zulegen. Wir haben ein Zukunftskonzept durchgesetzt und nicht nur Besitzstände verteidigt. Die Verbesserungen greifen direkt dort, wo die Menschen arbeiten, und unterstützen sie in ihrem täglichen betrieblichen Alltag. Zudem ist der Tarifvertrag nicht das Ergebnis irgendwelcher Verhandlungen im Hinterzimmer. Er wurde mit den Beschäftigten gemeinsam entwickelt und - besonders wichtig - maßgeblich von ihnen gegen den Willen des Arbeitgebers durchgesetzt. Kurzum: Der Zukunftstarifvertrag hat die IG Metall bei Airbus weiter nach vorne gebracht und eine gute Grundlage für die weitere Gewerkschaftsarbeit im Betrieb bereitet.

\section{Ein Beispiel für andere?}

Natürlich kann man den Prozess des Zukunftstarifvertrages bei Airbus nicht einfach auf andere Firmen übertragen. Trotzdem: Wenn es der IG Metall in einer Firma, die einen Auftragsbestand bis über das Jahr 2017 hat, gelingt, für sichere und gute Arbeit zu mobilisieren und sogar erfolgreich zu Arbeitskampfmaßnahmen aufzurufen, dann wird deutlich, dass die Beschäftigten auch wieder für qualitative Themen der Tarifpolitik erreichbar sind. Dies gilt bei Airbus gerade auch für die Hochqualifizierten. Bei der Entstehung, der Durchsetzung und der Umsetzung des Tarifvertrages haben sie sich stärker als in der Vergangenheit eingebracht. Auch die hohe Identifikation bei den qualitativen Themen in der Tarifrunde 2012 der Metall- und Elektroindustrie unterstreicht diese These. Auch hier waren die Beschäftigten für die unbefristete Übernahme der Auszubildenden und eine Regulierung von Leiharbeit mobilisierbar. Wie man arbeitet, mit welchem Freiraum und wie gerecht es im Unternehmen zugeht - hier wollen die Beschäftigten mitentscheiden. Dies tarifpolitisch aufzunehmen - ob betrieblich oder in der Fläche - tut der IG Metall gut und macht sie stärker.

\section{AUTOR}

DANIEL FRIEDRICH, Tarifsekretär des IG Metall-Bezirks Küste und Verhandlungsführer beim Zukunftstarifvertrag Airbus.

daniel.friedrich@igmetall.de 